\title{
Outage Analysis of a Spectrum-Sharing Cognitive Relaying Network with Improved Spectral Efficiency
}

\author{
Edgar Eduardo Benítez Olivo, Diana Pamela Moya Osorio, Daniel Benevides da Costa, \\ and José Cândido Silveira Santos Filho
}

\begin{abstract}
The outage performance of a spectrally efficient scheme for diversity exploitation in multiuser cognitive relaying networks under spectrum sharing constraints is investigated. In our analysis, we consider a secondary network composed by one source node communicating with one out of $L$ available destinations, which is selected according to the channel quality of the direct links. If the transmission through the selected direct link drops below a given threshold, a half-duplex decode-andforward relaying transmission is then invoked. For this purpose, it is first selected a new secondary destination so as to maximize the signal-to-noise ratio resulting from employing a maximal-ratio combining technique on the received signals from the secondary source and relay. In addition, assuming an underlay spectrumsharing approach, the overall transmit power at the secondary network is considered to be limited by the maximum tolerable interference power at the primary user receiver, as well as by the maximum transmit power available at the secondary nodes. An exact analytical expression for the outage probability of the proposed scheme is derived and validated by Monte Carlo simulations. Furthermore, closed-form expressions derived from the asymptotic analysis of the outage probability reveal that the proposed scheme achieves full diversity order, equal to $L+1$.
\end{abstract}

Keywords-Cognitive relaying networks, cooperative diversity, outage probability, underlay spectrum sharing.

\section{INTRODUCTION}

Spectrum sharing in cognitive radio networks is proposed as a promising approach that enables an efficient use of scarce radio resources [1], [2]. In an underlay spectrum sharing scenario, secondary users (SUs) are allowed to opportunistically and dynamically access a licensed band owned by primary users (PUs), as long as the resulting interference on the PUs is maintained below a given threshold. Cooperative relaying is another promising technique, which creates and exploits a new form of spatial diversity by leveraging the distributed signal processing and transmission from collaborating nodes of the network [3]. Both techniques have recently received great attention from the research community, once their joint use can achieve significantly improved outage performance and spectral efficiency [4], [5]. Due to this fact, several papers have investigated the performance of cognitive relaying networks

E. E. B. Olivo, D. P. M. Osorio, and J. C. S. Santos Filho are with the Department of Communications, School of Electrical and Computer Engineering, University of Campinas (UNICAMP), Campinas-SP, Brazil, Emails: \{ebenitez, dianamo, candido\}@ decom.fee.unicamp.br.

D. B. da Costa is with the Department of Computer Engineering, Federal University of Ceará (UFC), Sobral-CE, Brazil, E-mail: danielbcosta@ieee.org. in a multiuser scenario ${ }^{1}$ (see, for instance, [6], [7] and the references therein). In [6], Yang et al. proposed a cooperative relaying scheme to select the best available destination for communicating with the source under the aid of a half-duplex amplify-and-forward (AF) relay. However, because in [6] the direct links have not been considered and due to the halfduplex relay nature, the resulting diversity order and spectral efficiency were not satisfactory. In order to alleviate these problems, in [7], the authors considered the opportunistic use of the direct links in a multiuser cooperative scheme. Essentially, in that work, if the selected direct link is sufficiently good, then the relaying transmission is not employed, thus improving the overall spectral efficiency.

In this work, we extend the analysis of spectrally efficient schemes for a cognitive relaying network context. More specifically, employing a spectrally efficient scheme for spatialdiversity exploitation, we examine the outage performance of multiuser cognitive relaying networks under spectrum sharing constraints. In our analysis, we consider a secondary network composed by one source node communicating with the best one out of $L$ available destinations, which is selected according to the channel quality of the direct links. According to this scheme, if the transmission through the selected direct link drops below a given threshold, a half-duplex decode-andforward (DF) relaying transmission is carried out. For this purpose, it is first selected a new secondary destination that will maximize the signal-to-noise ratio resulting from employing a maximal-ratio combining (MRC) technique on the received signals from the SU source and SU relay. In addition, assuming an underlay spectrum-sharing approach, the overall transmit power at the secondary network is considered to be limited by the maximum tolerable interference power at the primary user receiver, as well as by the maximum transmit power available at the secondary nodes. An exact analytical expression for the outage probability of the proposed scheme is derived and validated by Monte Carlo simulations. Furthermore, closedform expressions derived from the asymptotic analysis of the outage probability reveal that the achievable diversity order equals $L+1$, thus not being affected by the interference temperature. By contrast, as shall be seen, the interference temperature causes the outage probability to "saturate" at a certain level.

\footnotetext{
${ }^{1}$ As well-known, multiuser diversity (MUD) is an inherent property of multiuser networks which can be exploited by opportunistically selecting the user with the best channel conditions.
} 


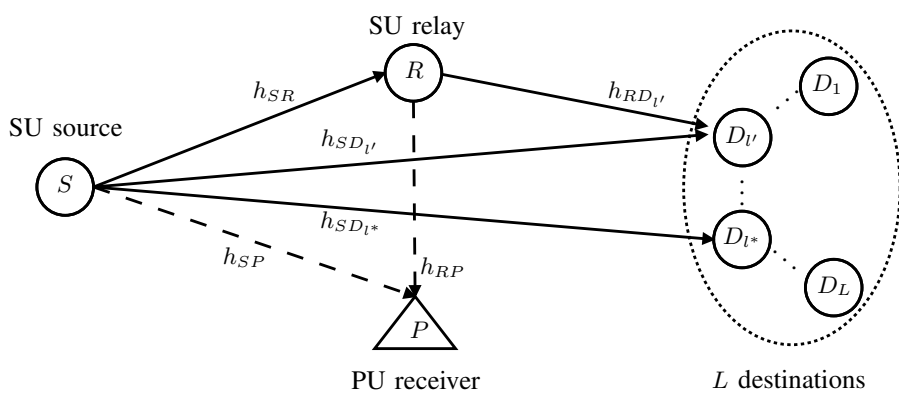

Fig. 1. System model (data link: solid line; interference link: dashed line).

The remainder of this paper is organized as follows. In Section II, the system model as well as the proposed spectrallyefficient scheme are described. Section III investigates the outage performance of the system under study, in which an exact analytical expression for the outage probability is derived. Based on this expression, an asymptotic analysis is carried out and the system diversity order is attained. Section IV presents some representative numerical results and insightful discussions are drawn. Finally, Section V concludes the paper.

In what follows, $f_{Z}(\cdot)$ denotes the probability density function (PDF) of a generic random variable $Z, E[\cdot]$ denotes expectation, and $\operatorname{Pr}(\cdot)$ denotes probability.

\section{System MODEL}

Consider a spectrum-sharing cognitive relaying network as shown in Fig. 1, which consists of one SU source $S$, one decode-and-forward SU relay $R, L$ SU destinations $D_{l}(l=$ $1, \ldots, L)$, and one PU receiver $P$. The solid lines denote the data links and the dashed lines denote the interference links. We assume that all nodes in the system are single-antenna devices operating in a half-duplex mode and on a time-division multiple access basis. Furthermore, we assume that the direct links between $S$ and $D_{l}$ are accessible and can be used to transmit information. In addition, the channels pertaining to the links between any two nodes, that is, the direct links, the link between $S$ and $R$, and the links from $R$ to $D_{l}$, are supposed to undergo independent but not necessarily identically distributed Rayleigh block fading. Hereafter, we denote the instantaneous channel coefficient and the distance between two arbitrary nodes $V$ and $W$ as $h_{V W}$ and $d_{V W}$, respectively, with $V W \in\left\{S R, S D_{l}, R D_{l}\right\}$. Moreover, by considering an underlay spectrum sharing scenario, let $I$ be the maximum tolerable interference power at the PU receiver, $P_{S}$ and $P_{R}$ be the maximum transmit powers at the SU source and SU relay, respectively, and assume that the background noise at all nodes is an additive white Gaussian noise (AWGN) with mean power $N_{0}$. Accordingly, the transmit powers at $S$ and $R$ can be written, respectively, as $\bar{P}_{S}=\min \left\{I /\left|h_{S P}\right|^{2}, P_{S}\right\}$ and $\bar{P}_{R}=\min \left\{I /\left|h_{R P}\right|^{2}, P_{R}\right\}$.

In this work, we make use of the spectrally-efficient improved incremental DF relaying scheme with MUD (MU-
IIDF) ${ }^{2}$ proposed in [7]. According to this scheme, the SU destination with the best channel quality of the direct link, $D_{l^{*}}$, is first selected among the $L$ available destinations, that is, $l^{*}=\arg \max _{l}\left\{\gamma_{S D_{l}}\right\}$, where $\gamma_{S D_{l}}=$ $\min \left\{I /\left|h_{S P}\right|^{2}, P_{S}\right\} d_{S D_{l}}^{-\alpha}\left|h_{S D_{l}}\right|^{2} / N_{0}^{l}$ is the instantaneous received signal-to-noise ratio (SNR) of the link between $S$ and $D_{l}$, with $\alpha$ being the pathloss exponent. Afterwards, the information transmission between $S$ and $D_{l^{*}}$ is carried out in one or two time slots, depending on the value of $\gamma_{S D_{l^{*}}}$. More specifically, during the first time slot, $S$ broadcasts information with transmit power $\bar{P}_{S}$, while $R, D_{l^{*}}$, as well as all other destinations $D_{l}\left(\forall l \neq l^{*}\right)$ listen to the transmission. For a given target spectral efficiency $\Re$ (bits/s/Hz), if $D_{l^{*}}$ is able to correctly decode the received information, that is, if $\log _{2}\left(1+\gamma_{S D_{l^{*}}}\right) \geq \Re$, or in an equivalent manner, if $\gamma_{S D_{l^{*}}} \geq 2^{\Re}-1$, it will broadcast a "success" message back to $S$ and $R$. Then, $S$ will be able to send new information during the next time slot, thus yielding an improved spectral efficiency. Otherwise, $D_{l^{*}}$ will broadcast a "failure" message back to $S$ and $R$, and the relaying transmission will be invoked in the second time slot. However, for this transmission, in order to take full advantage from the signals coming from the direct and relaying links and improve the transmission reliability against fading, an opportunistic scheduling mechanism is performed, which selects a new destination $D_{l^{\prime}}$ (possibly different from $\left.D_{l^{*}}\right)$ so as to maximize the SNR of the MRC operation of the source and relay signals, that is, $l^{\prime}=\arg \max _{l}\left\{\gamma_{S D_{l}}+\gamma_{R D_{l}}\right\}^{3}$. Subsequently, during the second time slot, $R$ will try to decode the foregoing information from $S$ and retransmit it to $D_{l^{\prime}}$ with transmit power $\bar{P}_{R}$. Afterwards, a MRC operation is executed at $D_{l^{\prime}}$ to combine the received signals from $S$ and $R$. Thus, the maximal mutual information $\mathcal{I}$ for the MU-IIDF scheme can be expressed as

$$
\mathcal{I}=\left\{\begin{array}{l}
\log _{2}\left(1+\gamma_{S D_{l^{*}}}\right), \text { if } \log _{2}\left(1+\gamma_{S D_{l^{*}}}\right) \geq \Re \\
\frac{1}{2} \log _{2}\left(1+\min \left\{\gamma_{S R}, \gamma_{S D_{l^{\prime}}}+\gamma_{R D_{l^{\prime}}}\right\}\right), \text { otherwise, }
\end{array}\right.
$$

where $\gamma_{S R}=\min \left\{I /\left|h_{S P}\right|^{2}, P_{S}\right\} d_{S R}^{-\alpha}\left|h_{S R}\right|^{2} / N_{0}$, and $\gamma_{R D_{l^{\prime}}}=$ $\min \left\{I /\left|h_{R P}\right|^{2}, P_{S}\right\} d_{R D_{l^{\prime}}}^{-\alpha}\left|h_{R D_{l^{\prime}}}\right|^{2} / N_{0}$.

\section{OUtage Probability AnAlysis}

In this section, an exact analytical expression for the outage probability will be derived, from which an asymptotic analysis will be carried out. To the best of the authors' knowledge, the subsequent analysis has not been performed in the technical literature before.

\footnotetext{
${ }^{2}$ In [7], it is also introduced the incremental DF relaying scheme with MUD (MU-IDF). Although both MU-IDF and MU-IIDF are shown to be highly spectrally efficient schemes in the high-SNR regime, attaining an expected spectral efficiency equal to that one obtained by direct transmission, the MU-IIDF scheme outperforms the MU-IDF scheme in terms of the outage probability. Thus, in this paper, the MU-IIDF is considered for analysis.

${ }^{3}$ In the case of MU-IDF scheme, this step is not performed. Instead of that, during the second time slot, $R$ will try to decode the information previously broadcast by $S$ and retransmit it to $D_{l^{*}}$, which was the destination initially selected. Then, it will combine the received signals by employing a maximalratio combining technique. However, $D_{l^{*}}$ may not be the optimal choice at that moment. Precisely, the MU-IIDF scheme attempts to circumvent this by selecting the destination $D_{l^{\prime}}$ that maximizes the resulting SNR of the MRC reception.
} 


\section{A. Exact Analysis}

For the considered system, an outage event occurs when both the direct transmission and the relaying transmission fail. Let $X \triangleq\left|h_{S P}\right|^{2}, Y \triangleq\left|h_{R P}\right|^{2}$, and $\tau \triangleq 2^{\Re}-1$. The conditional outage probability for a certain threshold $\tau$ can be formulated as

$$
\begin{array}{r}
P_{\text {out }}(\tau \mid X, Y)=\underbrace{\operatorname{Pr}\left(\gamma_{S D_{l^{*}}}<\tau, \gamma_{S R}<\tau \mid X\right)}_{K_{1}} \\
+\underbrace{\operatorname{Pr}\left(\gamma_{S D_{l^{*}}}<\tau, \gamma_{S R}>\tau, \gamma_{S D_{l^{\prime}}}+\gamma_{R D_{l^{\prime}}}<\tau \mid X, Y\right)}_{K_{2}},
\end{array}
$$

where, due to the statistical independence among the direct, first- and second-hop relaying links, and because of all of these links follow an exponential distribution according to the system model described above, $K_{1}$ can be calculated as

$$
\begin{aligned}
K_{1} & =\operatorname{Pr}\left(\gamma_{S D_{l^{*}}}<\tau \mid X\right) \operatorname{Pr}\left(\gamma_{S R}<\tau \mid X\right) \\
& =\prod_{l=1}^{L}\left(1-e^{-\tau \beta_{S D_{l}}}\right)\left(1-e^{-\tau \beta_{S R}}\right),
\end{aligned}
$$

with $\beta_{S D_{l}} \triangleq 1 / E\left[\gamma_{S D_{l}}\right]$ and $\beta_{S R} \triangleq 1 / E\left[\gamma_{S R}\right]$. In addition, since the event $\left\{\gamma_{S D_{l^{\prime}}}+\gamma_{R D_{l^{\prime}}}<\tau\right\}$ implies the event $\left\{\gamma_{S D_{l^{*}}}<\right.$ $\tau\}, K_{2}$ can be rewritten as

$$
K_{2}=\operatorname{Pr}\left(\gamma_{S R}>\tau \mid X\right) \underbrace{\operatorname{Pr}\left(\gamma_{S D_{l^{\prime}}}+\gamma_{R D_{l^{\prime}}}<\tau \mid X, Y\right)}_{K_{3}},
$$

where $\operatorname{Pr}\left(\gamma_{S R}>\tau \mid X\right)=\exp \left(-\tau \beta_{S R}\right)$, and $K_{3}$ is given by

$$
\begin{aligned}
K_{3} & =\operatorname{Pr}\left(\max _{l}\left\{\gamma_{S D_{l}}+\gamma_{R D_{l}}\right\}<\tau \mid X, Y\right) \\
& =\prod_{l=1}^{L} \underbrace{\operatorname{Pr}\left(\gamma_{S D_{l}}+\gamma_{R D_{l}}<\tau \mid X, Y\right)}_{\Phi_{l}},
\end{aligned}
$$

in which $\Phi_{l}$ can be derived as

$$
\begin{aligned}
\Phi_{l} & =\int_{0}^{\tau} \operatorname{Pr}\left(\gamma_{R D_{l}}<\tau-z \mid X, Y\right) f_{\gamma_{S D_{l}}}(z) d z \\
& =\int_{0}^{\tau}\left[1-e^{-(\tau-z) \beta_{R D_{l}}}\right] \beta_{S D_{l}} e^{-z \beta_{S D_{l}}} d z \\
& =\frac{\beta_{S D_{l}}\left(1-e^{-\tau \beta_{R D_{l}}}\right)-\beta_{R D_{l}}\left(1-e^{-\tau \beta_{S D_{l}}}\right)}{\beta_{S D_{l}}-\beta_{R D_{l}}},
\end{aligned}
$$

with $\beta_{R D_{l}} \triangleq 1 / E\left[\gamma_{R D_{l}}\right]$. Thus, unconditioning (1) w.r.t. $X$ and $Y$, the outage probability can be obtained by solving the following integral:

$$
P_{\text {out }}=\int_{0}^{\infty} \int_{0}^{\infty}\left[K_{1}(\tau \mid X)+K_{2}(\tau \mid X, Y)\right] f_{X}(x) f_{Y}(y) d x d y,
$$

where $K_{1}(\tau \mid X)$ and $K_{2}(\tau \mid X, Y)$ are given by (2) and (3), respectively. Furthermore, it is clear that

$$
\begin{aligned}
& \min \left(\frac{I}{X}, P_{S}\right)= \begin{cases}P_{S}, & \text { if } X \leq I / P_{S} \\
I / X, & \text { if } X>I / P_{S}\end{cases} \\
& \min \left(\frac{I}{Y}, P_{R}\right)= \begin{cases}P_{R}, & \text { if } Y \leq I / P_{R} \\
I / Y, & \text { if } Y>I / P_{R}\end{cases}
\end{aligned}
$$

Without loss of generality, let $P_{S}=P_{R}=Q$. Therefore, the outage probability in (6) can be split according to the four combined cases in (7) and (8) as $P_{\text {out }}=A+B+C+D$, where

$$
\begin{aligned}
& A=\int_{0}^{I / Q} \int_{0}^{I / Q}\left[K_{1}(\tau \mid X)+K_{2}(\tau \mid X, Y)\right] f_{X}(x) f_{Y}(y) d x d y \\
& B=\int_{I / Q}^{\infty} \int_{0}^{I / Q}\left[K_{1}(\tau \mid X)+K_{2}(\tau \mid X, Y)\right] f_{X}(x) f_{Y}(y) d x d y \\
& C=\int_{0}^{I / Q} \int_{I / Q}^{\infty}\left[K_{1}(\tau \mid X)+K_{2}(\tau \mid X, Y)\right] f_{X}(x) f_{Y}(y) d x d y \\
& D=\int_{I / Q}^{\infty} \int_{I / Q}^{\infty}\left[K_{1}(\tau \mid X)+K_{2}(\tau \mid X, Y)\right] f_{X}(x) f_{Y}(y) d x d y .(12)
\end{aligned}
$$

Making the appropriate substitutions in (9), we have that

$$
\begin{aligned}
A= & \int_{0}^{I / Q} \int_{0}^{I / Q} \prod_{l=1}^{L}\left(1-e^{-\tau \beta_{S D_{l}}^{Q}}\right)\left(1-e^{-\tau \beta_{S R}^{Q}}\right) \beta_{S P} e^{-x \beta_{S P}} \\
& \times \beta_{R P} e^{-y \beta_{R P}} d x d y+\int_{0}^{I / Q I / Q} \int_{0}^{-\tau \beta_{S R}^{Q} K_{3}^{Q}(\tau)} \\
& \times \beta_{S P} e^{-x \beta_{S P}} \beta_{R P} e^{-y \beta_{R P}} d x d y
\end{aligned}
$$

where $\beta_{S P} \triangleq 1 / E[X], \beta_{R P} \triangleq 1 / E[Y]$, and

$$
\beta_{V W}^{Q} \triangleq \frac{1}{E\left[Q d_{V W}^{-\alpha}\left|h_{V W}\right|^{2} / N_{0}\right]},
$$

with $V W \in\left\{S R, S D_{l}, R D_{l}\right\}$, and $K_{3}^{Q}(\tau)$ is given by (4), with $\beta_{S D_{l}}$ and $\beta_{R D_{l}}$ being defined as in (14). After some algebraic manipulations, (13) can be simplified to

$$
\begin{aligned}
A= & {\left[\prod_{l=1}^{L}\left(1-e^{-\tau \beta_{S D_{l}}^{Q}}\right)\left(1-e^{-\tau \beta_{S R}^{Q}}\right)+e^{-\tau \beta_{S R}^{Q}} K_{3}^{Q}(\tau)\right] } \\
& \times\left(1-e^{-\frac{I}{Q} \beta_{S P}}\right)\left(1-e^{-\frac{I}{Q} \beta_{R P}}\right)
\end{aligned}
$$

Similarly, after the appropriate substitutions, (10) can be expressed in a single-integral form as

$$
\begin{aligned}
& B=e^{-\frac{I}{Q} \beta_{R P}}\left(1-e^{-\tau \beta_{S R}^{Q}}\right)\left(1-e^{-\frac{I}{Q} \beta_{S P}}\right) \prod_{l=1}^{L}\left(1-e^{-\tau \beta_{S D_{l}}^{Q}}\right) \\
& +e^{-\tau \beta_{S R}^{Q}}\left(1-e^{-\frac{I}{Q} \beta_{S P}}\right) \int_{I / Q}^{\infty} K_{3}^{I}(\tau \mid Y) \beta_{R P} e^{-y \beta_{R P}} d y
\end{aligned}
$$

where $K_{3}^{I}(\tau \mid Y)$ is given by (4), with $\beta_{S D_{l}}$ and $\beta_{R D_{l}}$ being respectively defined as in (14) and

$$
y \beta_{R D_{l}}^{I} \triangleq \frac{1}{E\left[(I / y) d_{R D_{l}}^{-\alpha}\left|h_{R D_{l}}\right|^{2} / N_{0}\right]} .
$$


Following the same procedure in (11), it yields

$$
\begin{aligned}
& C=\left(1-e^{-\frac{I}{Q} \beta_{R P}}\right) \\
& \times \underbrace{\int_{I / Q}^{\infty} \prod_{l=1}^{L}\left(1-e^{-x \tau \beta_{S D_{l}}^{I}}\right)\left(1-e^{-x \tau \beta_{S R}^{I}}\right) \beta_{S P} e^{-x \beta_{S P}} d x}_{K_{4}} \\
& +\left(1-e^{-\frac{I}{Q} \beta_{R P}}\right) \int_{I / Q}^{\infty} e^{-x \tau \beta_{S R}^{I}} K_{3}^{I}(\tau \mid X) \beta_{S P} e^{-x \beta_{S P}} d x,
\end{aligned}
$$

where

$$
x \beta_{S W}^{I} \triangleq \frac{1}{E\left[(I / x) d_{S W}^{-\alpha}\left|h_{S W}\right|^{2} / N_{0}\right]},
$$

with $W \in\left\{D_{l}, R\right\}$, and $K_{3}^{I}(\tau \mid X)$ is given by (4), with $\beta_{R D_{l}}$ and $\beta_{S D_{l}}$ being respectively defined as in (14) and (19). After some algebraic manipulations, the first integral in (18) can be developed as

$$
\begin{aligned}
K_{4} \stackrel{(a)}{=} & \int_{I / Q}^{\infty} \sum_{l=0}^{L} \sum_{\substack{S_{l} \subseteq\{1, \ldots, L\} \\
\left|S_{l}\right|=l}}(-1)^{l} e^{-\sum_{j \in S_{l}} x \tau \beta_{S D_{j}}^{I}} \\
& \times\left(1-e^{\left.-x \tau \beta_{S R}^{I}\right)} \beta_{S P} e^{-x \beta} d x\right. \\
= & \sum_{l=0}^{L} \sum_{\substack{S_{l} \subseteq\{1, \ldots, L\} \\
\left|S_{l}\right|=l}}(-1)^{l} \beta_{S P}\left\{\frac{e^{-\frac{I}{Q}\left(\beta_{S P}+\tau \sum_{j \in S_{l}} \beta_{S D_{j}}^{I}\right)}}{\beta_{S P}+\tau \sum_{j \in S_{l}} \beta_{S D_{j}}^{I}}\right. \\
& \left.-\frac{e^{-\frac{I}{Q}\left[\beta_{S P}+\tau\left(\beta_{S R}^{I}+\sum_{j \in S_{l}} \beta_{S D_{j}}^{I}\right)\right]}}{\beta_{S P}+\tau\left(\beta_{S R}^{I}+\sum_{j \in S_{l}} \beta_{S D_{j}}^{I}\right)}\right\}
\end{aligned}
$$

where in step (a) we have made use of the multinomial expansion identity [9, eq. (33)]. Thus, (18) is also obtained in a single-integral form.

Following again the same procedure, (12) can be written as

$$
\begin{aligned}
& D=e^{-\frac{I}{Q} \beta_{R P}} K_{4} \\
& +\int_{I / Q}^{\infty} \int_{I / Q}^{\infty} e^{-x \tau \beta_{S R}^{I}} K_{3}^{I}(\tau \mid X, Y) \beta_{S P} e^{-x \beta_{S P}} \beta_{R P} e^{-y \beta_{R P}} d x d y,
\end{aligned}
$$

where $K_{3}^{I}(\tau \mid X, Y)$ is given by (4), with $\beta_{R D_{l}}$ and $\beta_{S D_{l}}$ being respectively defined as in (17) and (19).

Finally, using (15), (16), (18), and (21) into $P_{\text {out }}=A+B+$ $C+D$, we arrive at a new useful expression for the outage probability of the considered system. It is noteworthy that the single-fold integrals in (16) and (18), as well as the two-fold integral in (21), can be fast evaluated by numerical methods found in standard computing softwares, such as Matlab and Mathematica. Also, as far as the authors are aware, such analysis is new and it arises as a benchmark for further investigations in the design of spectrally efficient schemes for multiuser cognitive relaying networks.

\section{B. Asymptotic Analysis}

With the aim of gaining a better understanding of the outage performance of the system under study, as well as of its achievable diversity order, an asymptotic analysis of the outage behavior in the high-SNR regime is provided next ${ }^{4}$. By defining $\bar{\gamma} \triangleq 1 / N_{0}$ as the system SNR, and from the system model detailed in Section II, we can notice that $\beta_{S D_{l}}, \beta_{S R}$, and $\beta_{R D_{l}}$ go to zero as $\bar{\gamma} \rightarrow \infty$. In addition, by invoking the Maclaurin series expansion of the exponential function [9, eq. $(0.318 .2)$, we have that $e^{-x} \simeq 1-x$ when $x \rightarrow 0$. Using this, and after some tedious algebraic manipulations, the following asymptotic expressions can be respectively attained in a closed-form for the terms $A, B, C$, and $D$ in (15), (16), (18), and (21):

$$
\begin{aligned}
P_{\mathrm{out}}^{A} \simeq & \left\{\tau^{L+1} \beta_{S R}^{Q}\left(\prod_{l=1}^{L} \beta_{S D_{l}}^{Q}\right)+\left(\frac{\tau^{2}}{2}\right)^{L}\left(\prod_{l=1}^{L} \beta_{S D_{l}}^{Q} \beta_{R D_{l}}^{Q}\right)\right\} \\
& \times\left(1-e^{-\frac{I}{Q} \beta_{S P}}\right)\left(1-e^{-\frac{I}{Q} \beta_{R P}}\right) \propto\left(\frac{1}{\bar{\gamma}}\right)^{L+1} \\
P_{\mathrm{out}}^{B} \simeq & \tau^{L+1} \beta_{S R}^{Q} e^{-\frac{I}{Q} \beta_{R P}}\left(1-e^{-\frac{I}{Q} \beta_{S P}}\right)\left(\prod_{l=1}^{L} \beta_{S D_{l}}^{Q}\right) \\
& +\left(1-e^{-\frac{I}{Q} \beta_{S P}}\right)\left(\frac{\tau^{2}}{2}\right)^{L}\left(\prod_{l=1}^{L} \beta_{S D_{l}}^{Q} \beta_{R D_{l}}^{I}\right) \\
& \times \frac{1}{\beta_{R P}{ }^{L}} \Gamma\left(L+1, \frac{I}{Q} \beta_{R P}\right) \propto\left(\frac{1}{\bar{\gamma}}\right)^{L+1} \\
P_{\mathrm{out}}^{C} \simeq & \left(1-e^{-\frac{I}{Q} \beta_{R P}}\right)\left\{\tau^{L+1} \beta_{S R}^{I}\left(\prod_{l=1}^{L} \beta_{S D_{l}}^{I}\right) \frac{1}{\beta_{S P}{ }^{L+1}}\right. \\
& \times\left(\frac{1}{\bar{\gamma}}\right)^{L+1}, \\
& \times \Gamma\left(L+2, \frac{I}{Q} \beta_{S P}\right)+\left(\frac{\tau^{2}}{2}\right)^{L}\left(\prod_{l=1}^{L} \beta_{S D_{l}}^{I} \beta_{R D_{l}}^{Q}\right) \\
P_{\mathrm{out}}^{D} \simeq & \times \frac{1}{\beta_{S P}{ }^{L} \Gamma(L+1} \beta_{S R}^{I} e^{-\frac{I}{Q} \beta_{R P}}\left(\prod_{l=1}^{L} \beta_{S D_{l}}^{I}\right) \frac{1}{\beta_{S P}{ }^{L+1}} \\
& \left.\left.\times \frac{I}{Q} \beta_{S P}\right)\right\} \propto\left(\frac{1}{\bar{\gamma}}\right)^{L+1}
\end{aligned}
$$

where $\Gamma(\cdot, \cdot)$ denotes the upper incomplete gamma function $[9$, eq. (8.350.2)]. From the expressions above, and noting that $\beta_{V W}^{Q}$ and $\beta_{V W}^{I}$, with $V W \in\left\{S R, S D_{l}, R D_{l}\right\}$, are all inversely proportional to $\bar{\gamma}$, it can be seen that $P_{\text {out }} \propto(1 / \bar{\gamma})^{L+1}$, that is, the system attains a full diversity order, which equals $L+1$, with $L$ being the number of SU destinations. In addition, it is noteworthy that the diversity order is not impaired by the interference temperature constraint.

\section{NumericAl RESUlts AND Discussions}

In this section, we evaluate the outage performance of the considered cognitive relaying network by applying our

${ }^{4}$ It is worth mentioning that the asymptotic analysis is carried out in a direct manner on the integrand terms encountered in the outage probability expression. 


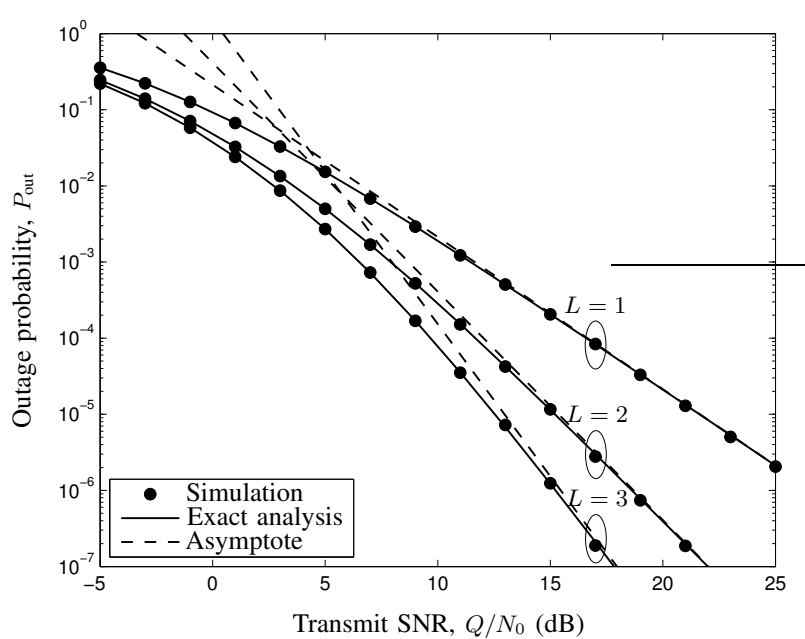

Fig. 2. Outage probability for different numbers of SU destinations $(I=$ $Q=0.5)$.

new analytical expressions to some representative examples. Monte Carlo simulation results are also provided in order to corroborate our formulas. For illustration purposes, we consider a 2-D plane network, where the SU source and the SU relay are located at $(0,0)$ and $(0.5,0)$, respectively, the SU destinations are clustered and collocated at $(1,0)$, and the PU is located at $(0,1)$. As a consequence, we have that $\beta_{S D_{l}}=\beta_{S D}$ and $\beta_{R D_{l}}=\beta_{R D}, \forall l$. Moreover, without loss of generality, we assume that the average channel gain between any two nodes is determined by the distance between them, and we set the target spectral efficiency $\Re$ to $1 \mathrm{bit} / \mathrm{s} / \mathrm{Hz}$ and the pathloss exponent $\alpha$ to 4 .

Fig. 2 shows the outage probability versus the maximum transmit SNR for different numbers of SU destinations. Specifically, we set $L=1,2,3$. As expected, as $L$ increases, the outage performance improves due to the MUD gain. Also, it is observed that the diversity order increases according to $L+1$.

Fig. 3 depicts the impact of the interference temperature constraint $I$ on the outage probability. As a sample example, we set the number of SU destinations $L$ to 2 . Owing to the interference constraint, the outage probability curves stabilize to a floor after the maximum allowed value of transmit $\mathrm{SNR}$ is achieved. Note that, as $I$ increases, higher values of transmit power are allowed and, as a consequence, the outage performance improves, approaching the no-constraint case.

In Figs. 2 and 3, it is noteworthy the excellent agreement between our exact analytical expressions (solid lines) and the simulation results (dots). Furthermore, in Fig. 2, note how our asymptotic expressions (dashed lines) provide a tight bound at moderate-to-high values of transmit SNR.

\section{Conclusions}

This paper studied the impact of the interference temperature constraints at a PU receiver on the outage performance of a multiuser cognitive relaying network. By using a spectrally efficient relaying scheme, an exact theoretical analysis for the outage probability was performed and validated by Monte Carlo simulations. In addition, closed-form expressions

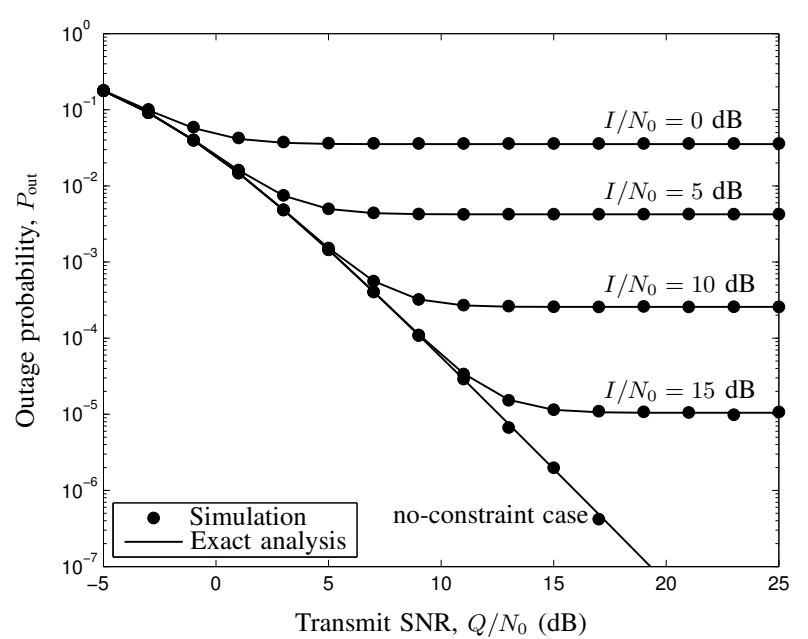

Fig. 3. Impact of the interference temperature constraint on the outage probability $(L=2)$.

derived from the asymptotic analysis at high SNR showed that the investigated system can achieve full diversity order, which is equal to $L+1$, and it was found that the diversity order is not affected by the interference temperature constraint. However, for a given interference constraint, the outage performance "saturates" after the maximum value of transmit power exceeds the maximum tolerable interference power.

\section{REFERENCES}

[1] J. Mitola and G. Q. Maguire, "Cognitive radio: Making software radios more personal,” IEEE Pers. Commun., vol. 6, no. 4, pp. 13-18, Aug. 1999.

[2] A. Goldsmith, S. Jafar, I. Maric, and S. Srinivasa, "Breaking spectrum gridlock with cognitive radios: An information theoretic perspective," Proc. IEEE, vol. 97, no. 5, pp. 894-914, May 2009.

[3] J. Lanemann, D. Tse, and G. Wornell, "Cooperative diversity in wireless networks: Efficient protocols and outage behavior," IEEE Trans. Inf. Theory, vol. 50, no. 12, pp. 3062-3080, Dec. 2004.

[4] S. Mishra, A. Sahai, and R. Brodersen, "Cooperative sensing among cognitive radios," in Proc. IEEE Int. Conf. Commun. (ICC '06), Istanbul, Turkey, Jun. 2006, pp. 1658-1663.

[5] L. Luo, P. Zhang, G. Zhang, and J. Qin, "Outage performance for cognitive relay networks with underlay spectrum sharing," IEEE Commun. Lett, vol. 15, no. 7, pp. 710-712, Jul. 2011.

[6] N. Yang, M. Elkashlan, and J. Yuan, "Outage probability of multiuser relay networks in Nakagami- $m$ fading channels," IEEE Trans. Veh. Technol., vol. 59, no. 5, pp. 2120-2132, Jun. 2010.

[7] H. Ding, J. Ge, D. B. da Costa, and Y. Guo, "Spectrally efficient diversity exploitation schemes for downlink cooperative cellular networks," IEEE Trans. Veh. Technol., vol. 61, no. 1, pp. 386-393, Jan. 2012.

[8] A. Papoullis, Probability, Random Variables, and Stochastic Processes, 4th edition. McGraw-Hill, 2002.

[9] I. Gradshteyn and I. Ryzhik, Table of Integrals, Series and Products, 7th edition. Academic Press, 2007. 